Bull. Korean Math. Soc. 46 (2009), No. 2, pp. 331-346

DOI 10.4134/BKMS.2009.46.2.331

\title{
EVALUATING SOME DETERMINANTS OF MATRICES WITH RECURSIVE ENTRIES
}

\author{
Ali Reza Moghaddamfar, Seyyed Navid Salehy, and Seyyed Nima Salehy
}

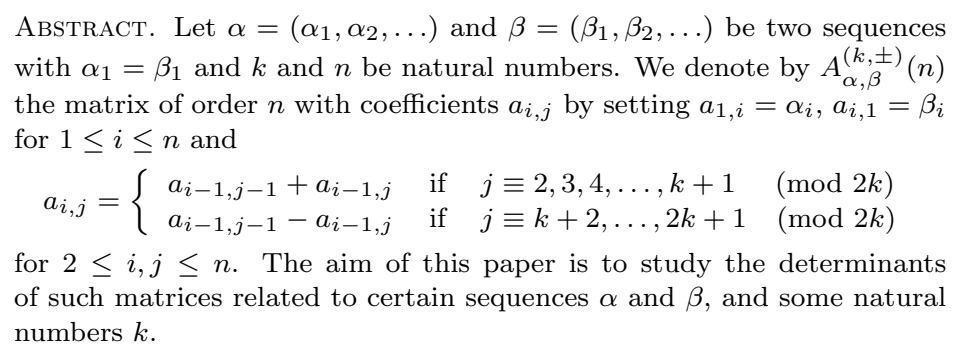

\section{Introduction}

In [1], R. Bacher considered the determinants of matrices associated to the Pascal triangle. Furthermore, he introduced the generalized Pascal triangles as follows. Let $\alpha=\left(\alpha_{1}, \alpha_{2}, \ldots\right)$ and $\beta=\left(\beta_{1}, \beta_{2}, \ldots\right)$ be two sequences starting with a common first term $\alpha_{1}=\beta_{1}$. Define a matrix $P_{\alpha, \beta}(n)$ of order $n$ with coefficients $p_{i, j}$ by setting $p_{i, 1}=\beta_{i}, p_{1, i}=\alpha_{i}$ for $1 \leq i \leq n$ and $p_{i, j}=p_{i-1, j}+$ $p_{i, j-1}$ for $2 \leq i, j \leq n$. The infinite matrix $P_{\alpha, \beta}(\infty)$ is called the generalized Pascal triangle associated to the sequences $\alpha$ and $\beta$. In addition he investigated some other similar constructions and made many interesting observations and posed some conjectures. Some of his conjectures were thoroughly investigated in [3] with positive answers.

In constructing the generalized Pascal triangles or the other similar constructions in which the coefficients, except for the first row and column, are determined by a recursive relation, only one recursive relation is used. Here we are willing to construct some similar arrangements associated to two arbitrary sequences $\alpha$ and $\beta$ being in the first row and column, respectively, and the remaining coefficients are determined by two different recursive relations. Let us define this more precisely as follows.

Received May 23, 2008.

2000 Mathematics Subject Classification. 11C20, 15A15, 15A36, 15A57.

Key words and phrases. determinant, LU-factorization, recurrence relation.

This research was in part supported by a grant from IPM (No. 84200039). 
Definition. Let $\alpha=\left(\alpha_{1}, \alpha_{2}, \ldots\right)$ and $\beta=\left(\beta_{1}, \beta_{2}, \ldots\right)$ be two sequences starting with a common first term $\alpha_{1}=\beta_{1}$ and $k$ be a natural number. Define a matrix $A_{\alpha, \beta}^{(k, \pm)}(n)$ of order $n$ with coefficients $a_{i, j}$ by setting $a_{i, 1}=\beta_{i}, a_{1, i}=\alpha_{i}$ for $1 \leq i \leq n$ and

$$
a_{i, j}=\left\{\begin{array}{lll}
a_{i-1, j-1}+a_{i-1, j} & \text { if } j \equiv 2,3,4, \ldots, k+1 \quad(\bmod 2 k) \\
a_{i-1, j-1}-a_{i-1, j} & \text { if } j \equiv k+2, \ldots, 2 k+1 \quad(\bmod 2 k)
\end{array}\right.
$$

for $2 \leq i, j \leq n$. When $k=1$, we put $A_{\alpha, \beta}^{ \pm}(n)=A_{\alpha, \beta}^{(1, \pm)}(n)$.

In general, we are interested in the sequence of the determinants

$$
\left(\operatorname{det} A_{\alpha, \beta}^{(k, \pm)}(1), \operatorname{det} A_{\alpha, \beta}^{(k, \pm)}(2), \ldots, \operatorname{det} A_{\alpha, \beta}^{(k, \pm)}(n), \ldots\right),
$$

where $\alpha$ and $\beta$ are certain sequences having a common first entry.

On the other hand, when we consider the constant sequence $\alpha=(1,1,1, \ldots)$, we notice that the generalized Pascal triangle $P_{\alpha, \alpha}(\infty)$ is, in fact, the classical Pascal triangle. Hence, in the early studies, we restrict our investigation to this sequence $\alpha=(1,1,1, \ldots)$ only, and we consider the principal minors of infinite matrices $A_{\alpha, \alpha}^{(k, \pm)}(\infty)$.

In this research, it has been tried to prove three theorems.

Theorem 1.1. The matrices $A_{\alpha, \alpha}^{ \pm}(n)$ associated to the sequence $\alpha=(1,1, \ldots)$ have the determinant $3^{\left[\frac{n-1}{2}\right]}$ for every natural number $n$. In other words, we have

$$
\operatorname{det} A_{\alpha, \alpha}^{ \pm}(n)= \begin{cases}3^{l-1} & \text { if } \quad n=2 l, \quad(l=1,2, \ldots) \\ 3^{l} & \text { if } \quad n=2 l+1 .(l=0,1,2, \ldots)\end{cases}
$$

Theorem 1.2. The sequence ( $\left.\operatorname{det} A_{\alpha, \alpha}^{(2, \pm)}(n)\right)$ of determinants associated to the sequence $\alpha=(1,1,1, \ldots)$ satisfies the following

$$
\operatorname{det} A_{\alpha, \alpha}^{(2, \pm)}(n)= \begin{cases}(-5)^{2 l-1} & \text { if } \quad n=4 l, \quad(l=1,2, \ldots) \\ (-5)^{2 l} & \text { if } \quad n=4 l+r .(r=1,2,3 ; l=0,1,2, \ldots)\end{cases}
$$

Theorem 1.3. The sequence ( $\left.\operatorname{det} A_{\alpha, \alpha}^{(3, \pm)}(n)\right)$ of determinants associated to the sequence $\alpha=(1,1,1, \ldots)$ satisfies the following

$$
\operatorname{det} A_{\alpha, \alpha}^{(3, \pm)}(n)=\left\{\begin{array}{lll}
11^{3 l-1} & \text { if } n=6 l, \quad(l=1,2, \ldots) \\
11^{3 l} & \text { if } n=6 l+r,(r=1,2,3,4 ; l=0,1,2, \ldots) \\
11^{3 l+1} & \text { if } n=6 l+5 .(l=0,1,2, \ldots)
\end{array}\right.
$$

Here, we have the following conjecture:

Conjecture. Let $k$ and $n$ be natural numbers and $n-1=r k+s$ for some $r, s$ with $r \geq 0$ and $0 \leq s<k$. Let $\alpha=(1,1,1, \ldots)$. Then we have

$$
\operatorname{det} A_{\alpha, \alpha}^{(k, \pm)}(n)= \begin{cases}\omega^{r k / 2} & \text { if } r \text { is even, } \\ \omega^{k(r-1) / 2+s} & \text { if } r \text { is odd, }\end{cases}
$$

where $\omega=\left[1-(-2)^{k+2}\right] / 3$. 


\section{Main results}

As we mentioned before, we should concentrate on the sequence of determinants

$$
\left(\operatorname{det} A_{\alpha, \alpha}^{(k, \pm)}(1), \operatorname{det} A_{\alpha, \alpha}^{(k, \pm)}(2), \ldots, \operatorname{det} A_{\alpha, \alpha}^{(k, \pm)}(n), \ldots\right)
$$

for certain $k$. Therefore, in order to start, we consider the case $k=1$, and prove the following theorem.

Theorem 1. The matrices $A_{\alpha, \alpha}^{ \pm}(n)$ associated to the sequence $\alpha=(1,1,1, \ldots)$ have the determinant $3^{\left[\frac{n-1}{2}\right]}$ for every natural number $n$.

Proof. We apply LU-factorization method (see [2]). We claim that

$$
A_{\alpha, \alpha}^{ \pm}(n)=L \cdot U
$$

where $L=A_{\beta, \alpha}^{ \pm}(n)$ with $\beta=(1,0,0,0, \ldots)$, and where

$$
U=\left[\begin{array}{c}
U_{1} \\
U_{2} \\
\vdots \\
U_{n}
\end{array}\right]
$$

with

$$
U_{i}= \begin{cases}\underbrace{(1,1,1, \ldots, 1}_{n \text { times }}) & \text { if } i=1, \\ (\underbrace{0,0, \ldots, 0}_{i-1 \text { times }}, \underbrace{1,-1,1,-1, \ldots, u_{i, n-1}, u_{i, n}}_{n-i+1 \text { times }(2-\text { periodic })}) & \text { if } i \stackrel{2}{\equiv} 0, \\ (\underbrace{0,0, \ldots, 0}_{i-1 \text { times }}, \underbrace{3,-1,3,-1,3, \ldots, u_{i, n-1}, u_{i, n}}_{n-i+1 \text { times }(2-\text { periodic })}) & \text { if } i>1 \text { and } i \stackrel{2}{\equiv} 1,\end{cases}
$$

and $\left(u_{i, n-1}, u_{i, n}\right)$ is satisfied in Table 1.

Table 1.

\begin{tabular}{|l|l|l|}
\hline$i \backslash n$ & $n \stackrel{2}{\equiv} 0$ & $n \stackrel{2}{\equiv} 1$ \\
\hline$i \stackrel{2}{\equiv} 0$ & $(-1,1)$ & $(1,-1)$ \\
\hline$i \stackrel{2}{\equiv} 1$ & $(3,-1)$ & $(-1,3)$ \\
\hline
\end{tabular}

The matrix $L$ is a lower triangular matrix with 1's on the diagonal, whereas $U$ is an upper triangular matrix with diagonal entries 


$$
\begin{array}{ll}
1 & \text { if } n=1, \\
1,1 & \text { if } n=2, \\
1, \underbrace{1,3,1,3,1,3, \ldots, 1,3}_{n-1 \text { times }(2-\text { periodic })} & \text { if } n>1 \text { and } n \stackrel{2}{=} 1, \\
1, \underbrace{1,3,1,3,1,3, \ldots, 3,1}_{n-1 \text { times }(2-\text { periodic })} & \text { if } n>2 \text { and } n \stackrel{2}{=} 0 .
\end{array}
$$

Since $\operatorname{det} L=1$ and $\operatorname{det} U=3^{\left[\frac{n-1}{2}\right]}$, it is obvious that the claimed factorization of $A_{\alpha, \alpha}^{ \pm}(n)$ immediately implies the validity of the theorem.

Suppose that

$$
L=\left(l_{i, j}\right)_{1 \leq i, j \leq n} \quad \text { and } \quad U=\left(u_{i, j}\right)_{1 \leq i, j \leq n} .
$$

Then by definition, we have $l_{1,1}=1, l_{1, j}=0, l_{i, 1}=1$ for $2 \leq i, j \leq n$ and

$$
l_{i, j}= \begin{cases}l_{i-1, j-1}+l_{i-1, j} & \text { if } j \stackrel{2}{\equiv} 0 \\ l_{i-1, j-1}-l_{i-1, j} & \text { if } j \stackrel{2}{\equiv} 1\end{cases}
$$

for $2 \leq i, j \leq n$. Also we have

$$
\left(u_{1, j}, u_{2, j}, \ldots, u_{n, j}\right)^{T}= \begin{cases}(1,0,0, \ldots, 0)^{T} & j=1, \\ (1, \underbrace{1,-1,1,-1, \ldots,-1,1}_{j-1 \text { times }(2-\text { periodic })}, \underbrace{0, \ldots, 0}_{n-j})^{T} & j \stackrel{2}{\equiv} 0, \\ (1, \underbrace{-1,3,-1,3, \ldots,-1,3}_{j-1 \text { times }(2-\text { periodic })}, \underbrace{0, \ldots, 0}_{n-j})^{T} & j \stackrel{2}{\equiv} 1 .\end{cases}
$$

For the proof of the claimed factorization we compute the $(i, j)$-entry of $L \cdot U$, that is

$$
(L \cdot U)_{i, j}=\sum_{k=1}^{n} l_{i, k} u_{k, j} .
$$

It is easy to see that it is enough to show that $(L \cdot U)_{1, j}=1,(L \cdot U)_{i, 1}=1$ for $1 \leq i, j \leq n$ and

$$
(L \cdot U)_{i, j}= \begin{cases}(L \cdot U)_{i-1, j-1}+(L \cdot U)_{i-1, j} & j \stackrel{2}{\equiv} 0 \\ (L \cdot U)_{i-1, j-1}-(L \cdot U)_{i-1, j} & j \stackrel{2}{\equiv} 1\end{cases}
$$

for $2 \leq i, j \leq n$, in order to prove the theorem.

Let us do the required calculations. First, suppose that $i=1$. Then

$$
(L \cdot U)_{1, j}=\sum_{k=1}^{n} l_{1, k} u_{k, j}=l_{1,1} u_{1, j}=1 .
$$

Next, suppose that $j=1$. In this case we obtain

$$
(L \cdot U)_{i, 1}=\sum_{k=1}^{n} l_{i, k} u_{k, 1}=l_{i, 1} u_{1,1}=1 .
$$


Finally, we assume that $2 \leq i, j \leq n$. We split the proof into two cases, according to the following possibilities for $j$.

Case 1. $j \stackrel{2}{\equiv} 0$. In this case we claim that

$$
(L \cdot U)_{i, j}=(L \cdot U)_{i-1, j-1}+(L \cdot U)_{i-1, j} \cdot
$$

Since $j-1 \stackrel{2}{\equiv} 1$, by $(2)$ we get

$$
(L \cdot U)_{i-1, j-1}=\sum_{k=1}^{n} l_{i-1, k} u_{k, j-1}=1-\sum_{k=1}^{\frac{j-2}{2}} l_{i-1,2 k}+3 \sum_{k=1}^{\frac{j-2}{2}} l_{i-1,2 k+1},
$$

and since $j \stackrel{2}{\equiv} 0$ we obtain

$$
(L \cdot U)_{i-1, j}=\sum_{k=1}^{n} l_{i-1, k} u_{k, j}=1+\sum_{k=1}^{\frac{j}{2}} l_{i-1,2 k}-\sum_{k=1}^{\frac{j-2}{2}} l_{i-1,2 k+1},
$$

and ultimately

$$
(L \cdot U)_{i-1, j-1}+(L \cdot U)_{i-1, j}=2+2 \sum_{k=1}^{\frac{j-2}{2}} l_{i-1,2 k+1}+l_{i-1, j} .
$$

Again, since $j \stackrel{2}{\equiv} 0$ we obtain

$$
(L \cdot U)_{i, j}=\sum_{k=1}^{n} l_{i, k} u_{k, j}=l_{i, 1}+\sum_{k=1}^{\frac{j}{2}} l_{i, 2 k}-\sum_{k=1}^{\frac{j-2}{2}} l_{i, 2 k+1}
$$

and by (1) we get

$$
(L \cdot U)_{i, j}=l_{i, 1}+\sum_{k=1}^{\frac{j}{2}}\left(l_{i-1,2 k-1}+l_{i-1,2 k}\right)-\sum_{k=1}^{\frac{j-2}{2}}\left(l_{i-1,2 k}-l_{i-1,2 k+1}\right),
$$

and after some further simplification we obtain

$$
(L \cdot U)_{i, j}=2+2 \sum_{k=1}^{\frac{j-2}{2}} l_{i-1,2 k+1}+l_{i-1, j}
$$

Now, from (6) and (7) we obtain (5).

Case 2. $j \stackrel{2}{\equiv} 1$. In this case we claim that

$$
(L \cdot U)_{i, j}=(L \cdot U)_{i-1, j-1}-(L \cdot U)_{i-1, j} .
$$

Here, since $j-1 \stackrel{2}{\equiv} 0$, by (2) we obtain

$$
(L \cdot U)_{i-1, j-1}=\sum_{k=1}^{n} l_{i-1, k} u_{k, j-1}=1+\sum_{k=1}^{\frac{j-1}{2}} l_{i-1,2 k}-\sum_{k=1}^{\frac{j-3}{2}} l_{i-1,2 k+1}
$$


and similarly we deduce that

$$
(L \cdot U)_{i-1, j}=\sum_{k=1}^{n} l_{i-1, k} u_{k, j}=1-\sum_{k=1}^{\frac{j-1}{2}} l_{i-1,2 k}+3 \sum_{k=1}^{\frac{j-1}{2}} l_{i-1,2 k+1},
$$

because $j \stackrel{2}{\equiv} 1$. Therefore by an easy calculation we conclude that

$$
(L \cdot U)_{i-1, j-1}-(L \cdot U)_{i-1, j}=2 \sum_{k=1}^{\frac{j-1}{2}} l_{i-1,2 k}-4 \sum_{k=1}^{\frac{j-3}{2}} l_{i-1,2 k+1}-3 l_{i-1, j} .
$$

Again, since $j \stackrel{2}{=} 1$ we have

$$
(L \cdot U)_{i, j}=\sum_{k=1}^{n} l_{i, k} u_{k, j}=l_{i, 1}-\sum_{k=1}^{\frac{j-1}{2}} l_{i, 2 k}+3 \sum_{k=1}^{\frac{j-1}{2}} l_{i, 2 k+1} .
$$

Now, by (1) we obtain

$$
(L \cdot U)_{i, j}=1-\sum_{k=1}^{\frac{j-1}{2}}\left(l_{i-1,2 k-1}+l_{i-1,2 k}\right)+3 \sum_{k=1}^{\frac{j-1}{2}}\left(l_{i-1,2 k}-l_{i-1,2 k+1}\right),
$$

and simply we can observe that

$$
(L \cdot U)_{i, j}=2 \sum_{k=1}^{\frac{j-1}{2}} l_{i-1,2 k}-4 \sum_{k=1}^{\frac{j-3}{2}} l_{i-1,2 k+1}-3 l_{i-1, j} .
$$

Now, from (9) and (10) we obtain (8).

Therefore, from (3), (4), (5) and (8) we conclude the theorem.

Next, we focus on the sequence $\left(\operatorname{det} A_{\alpha, \alpha}^{(2, \pm)}(n)\right)$ for $n \in \mathbb{N}$.

Theorem 2. The sequence ( $\left.\operatorname{det} A_{\alpha, \alpha}^{(2, \pm)}(n)\right)$ of determinants associated to the sequence $\alpha=(1,1,1, \ldots)$ satisfies the following

$$
\operatorname{det} A_{\alpha, \alpha}^{(2, \pm)}(n)= \begin{cases}(-5)^{2 l-1} & \text { if } n=4 l, \quad(l=1,2, \ldots) \\ (-5)^{2 l} & \text { if } \quad n=4 l+r .(r=1,2,3, l=0,1,2, \ldots)\end{cases}
$$

Proof. Again, we use the LU-factorization method. Here, we claim that

$$
A_{\alpha, \alpha}^{(2, \pm)}(n)=L \cdot U
$$

where $L=A_{\beta, \alpha}^{(2, \pm)}(n)$ with $\beta=(1,0,0, \ldots)$ and where

$$
U=\left[\begin{array}{c}
U_{1} \\
U_{2} \\
\vdots \\
U_{n}
\end{array}\right]
$$


with

$$
U_{i}= \begin{cases}\underbrace{(1,1,1, \ldots, 1}_{n \text { times }}) & i=1, \\ \underbrace{0,0, \ldots, 0}_{i=1 \text { times }}, \underbrace{-5,1,1,-1,-5,1,1,-1, \ldots, u_{i, n-1}, u_{i, n}}_{n-i+1 \text { times }(4-\text { periodic })}) & i \stackrel{4}{=} 0, \\ \underbrace{0,0, \ldots, 0}_{i-1 \text { times }}, \underbrace{-5,3,-1,-1,-5,3,-1,-1, \ldots, u_{i, n-1}, u_{i, n}}_{n-i+1 \text { times }(4-\text { periodic })}) & i \stackrel{4}{\equiv} 1, \\ (\underbrace{0,0, \ldots, 0}_{i-1 \text { times }}, \underbrace{1,1,-1,-1,1,1,-1,-1, \ldots, u_{i, n-1}, u_{i, n}}_{n-i+1 \text { times }(4-\text { periodic })}) & i \stackrel{4}{=} 2, \\ (\underbrace{0,0, \ldots, 0}_{i-1 \text { times }}, \underbrace{1,3,1,-1,1,3,1,-1, \ldots, u_{i, n-1}, u_{i, n}}_{n-i+1 \text { times }(4-\text { periodic })}) & i \stackrel{4}{\equiv} 3,\end{cases}
$$

and $\left(u_{i, n-1}, u_{i, n}\right)$ is satisfied in Table 2 .

Table 2.

\begin{tabular}{|l|l|l|l|l|}
\hline$i \backslash n$ & $n \stackrel{4}{\equiv} 0$ & $n \stackrel{4}{\equiv} 1$ & $n \stackrel{4}{\equiv} 2$ & $n \stackrel{\text { 帛 }}{=}$ \\
\hline$i \stackrel{4}{\equiv} 0$ & $(-1,-5)$ & $(-5,1)$ & $(1,1)$ & $(1,-1)$ \\
\hline$i \stackrel{4}{\equiv} 1$ & $(-1,-1)$ & $(-1,-5)$ & $(-5,3)$ & $(3,-1)$ \\
\hline$i \stackrel{4}{\equiv} 2$ & $(1,-1)$ & $(-1,-1)$ & $(-1,1)$ & $(1,1)$ \\
\hline$i \stackrel{4}{\equiv} 3$ & $(1,3)$ & $(3,1)$ & $(1,-1)$ & $(-1,1)$ \\
\hline
\end{tabular}

The matrix $L$ is a lower triangular matrix with 1's on the diagonal, whereas $U$ is an upper triangular matrix with diagonal entries

$$
1, \underbrace{1,1,-5,-5,1,1, \ldots, u_{n-1, n-1}, u_{n, n}}_{4-\text { periodic }},
$$

where

$$
\left(u_{n-1, n-1}, u_{n, n}\right)= \begin{cases}(1,-5) & \text { if } n \stackrel{4}{=} 0 \\ (-5,-5) & \text { if } n \stackrel{4}{=} 1 \\ (-5,1) & \text { if } n \stackrel{4}{\equiv} 2 \\ (1,1) & \text { if } n \stackrel{4}{\equiv} 3 .\end{cases}
$$

Since $\operatorname{det} L=1$ and

$$
\operatorname{det} U=\left\{\begin{array}{lll}
(-5)^{2 l-1} & \text { if } & n=4 l, \quad(l=1,2, \ldots) \\
(-5)^{2 l} & \text { if } & n=4 l+r .
\end{array}\right.
$$

Again, it is immediately obvious that the claimed factorization of $A_{\alpha, \alpha}^{(2, \pm)}(n)$ implies the validity of the theorem. 
Suppose that

$$
L=\left(l_{i, j}\right)_{1 \leq i, j \leq n} \quad \text { and } \quad U=\left(u_{i, j}\right)_{1 \leq i, j \leq n} .
$$

Then by definition, we have $l_{1,1}=1, l_{1, j}=0, l_{i, 1}=1$ for $2 \leq i, j \leq n$ and

$$
l_{i, j}= \begin{cases}l_{i-1, j-1}+l_{i-1, j} & \text { if } j \stackrel{4}{\equiv} 2,3 \\ l_{i-1, j-1}-l_{i-1, j} & \text { if } j \stackrel{4}{\equiv} 0,1\end{cases}
$$

for $2 \leq i, j \leq n$. Moreover, the $j$ th column of $U$ can be considered as follows:

$$
\left(u_{1, j}, \ldots, u_{n, j}\right)^{T}= \begin{cases}(1,0,0, \ldots, 0)^{T} & j=1, \\ (1, \underbrace{-1,3,-5,-1,-1,3,-5,-1, \ldots, 3,-5}_{j-1 \text { times }(4-\text { periodic })}, \underbrace{0, \ldots, 0}_{n-j})^{T} & j \stackrel{4}{\equiv} 0, \\ (1, \underbrace{-1,1,1,-5,-1,1,1,-5, \ldots, 1,-5}_{j-1 \text { times }(4-\text { periodic })}, \underbrace{0, \ldots, 0}_{n-j})^{T} & j \stackrel{4}{=} 1, \\ (1, \underbrace{1,-1,1,3,1,-1,1,3, \ldots, 3,1}_{j-1 \text { times }(4-\text { periodic })}, \underbrace{0, \ldots, 0}_{n-j})^{T} & j \stackrel{4}{\equiv} 2, \\ (1, \underbrace{1,1,-1,-1,1,1,-1,-1, \ldots, 1}_{j-1 \text { times }(4-\text { periodic })}, \underbrace{0, \ldots, 0}_{n-j})^{T} & j \stackrel{4}{\equiv} 3 .\end{cases}
$$

For the proof of the claimed factorization we need again some calculations. In fact, the $(i, j)$-entry of $L \cdot U$ is

$$
(L \cdot U)_{i, j}=\sum_{k=1}^{n} l_{i, k} u_{k, j} .
$$

It is easy to see that it is enough to show that $(L \cdot U)_{1, j}=1,(L \cdot U)_{i, 1}=1$ for $1 \leq i, j \leq n$ and

$$
(L \cdot U)_{i, j}= \begin{cases}(L \cdot U)_{i-1, j-1}+(L \cdot U)_{i-1, j} & j \stackrel{4}{\equiv} 2,3 \\ (L \cdot U)_{i-1, j-1}-(L \cdot U)_{i-1, j} & j \stackrel{4}{\equiv} 0,1\end{cases}
$$

for $2 \leq i, j \leq n$, in order to prove the theorem.

Again, we verify the claim by a direct calculation. First, suppose that $i=1$. Then

$$
(L \cdot U)_{1, j}=\sum_{k=1}^{n} l_{1, k} u_{k, j}=l_{1,1} u_{1, j}=1
$$

Next, suppose that $j=1$. In this case we obtain

$$
(L \cdot U)_{i, 1}=\sum_{k=1}^{n} l_{i, k} u_{k, 1}=l_{i, 1} u_{1,1}=1 .
$$

Finally, we assume that $2 \leq i, j \leq n$. We split the proof into four cases, according to the following possibilities for $j$. 
Case 1. $j \stackrel{4}{\equiv} 0$. In this case we claim that

$$
(L \cdot U)_{i, j}=(L \cdot U)_{i-1, j-1}-(L \cdot U)_{i-1, j} .
$$

Since $j-1 \stackrel{4}{=} 3$, we obtain

$(L \cdot U)_{i-1, j-1}=l_{i-1,1}-\sum_{k=1}^{\frac{j-4}{4}} l_{i-1,4 k}-\sum_{k=1}^{\frac{j-4}{4}} l_{i-1,4 k+1}+\sum_{k=0}^{\frac{j-4}{4}} l_{i-1,4 k+2}+\sum_{k=0}^{\frac{j-4}{4}} l_{i-1,4 k+3}$,

and since $j \stackrel{4}{\equiv} 0$, it follows that

$(L \cdot U)_{i-1, j}=l_{i-1,1}-5 \sum_{k=1}^{\frac{j}{4}} l_{i-1,4 k}-\sum_{k=1}^{\frac{j-4}{4}} l_{i-1,4 k+1}-\sum_{k=0}^{\frac{j-4}{4}} l_{i-1,4 k+2}+3 \sum_{k=0}^{\frac{j-4}{4}} l_{i-1,4 k+3} \cdot$

Consequently, we obtain

$$
(L \cdot U)_{i-1, j-1}-(L \cdot U)_{i-1, j}=4 \sum_{k=1}^{\frac{j-4}{4}} l_{i-1,4 k}+2 \sum_{k=0}^{\frac{j-4}{4}} l_{i-1,4 k+2}-2 \sum_{k=0}^{\frac{j-4}{4}} l_{i-1,4 k+3}-5 l_{i-1, j} .
$$

On the other hand since $j \stackrel{4}{\equiv} 0$, we get

$$
(L \cdot U)_{i, j}=l_{i, 1}-5 \sum_{k=1}^{\frac{j}{4}} l_{i, 4 k}-\sum_{k=1}^{\frac{j-4}{4}} l_{i, 4 k+1}-\sum_{k=0}^{\frac{j-4}{4}} l_{i, 4 k+2}+3 \sum_{k=0}^{\frac{j-4}{4}} l_{i, 4 k+3} .
$$

Now by (11) we deduce that

$$
\begin{aligned}
(L \cdot U)_{i, j}= & 1-5 \sum_{k=1}^{\frac{j}{4}}\left(l_{i-1,4 k-1}-l_{i-1,4 k}\right)-\sum_{k=1}^{\frac{j-4}{4}}\left(l_{i-1,4 k}-l_{i-1,4 k+1}\right) \\
& -\sum_{k=0}^{\frac{j-4}{4}}\left(l_{i-1,4 k+1}+l_{i-1,4 k+2}\right)+3 \sum_{k=0}^{\frac{j-4}{4}}\left(l_{i-1,4 k+2}+l_{i-1,4 k+3}\right),
\end{aligned}
$$

and after some further simplifications the expression reduces to

$$
(L \cdot U)_{i, j}=4 \sum_{k=1}^{\frac{j-4}{4}} l_{i-1,4 k}+2 \sum_{k=0}^{\frac{j-4}{4}} l_{i-1,4 k+2}-2 \sum_{k=0}^{\frac{j-4}{4}} l_{i-1,4 k+3}-5 l_{i-1, j} .
$$

Now, from (16) and (17) we obtain (15).

Case 2. $j \stackrel{4}{\equiv} 1$. Here, we claim that

$$
(L \cdot U)_{i, j}=(L \cdot U)_{i-1, j-1}-(L \cdot U)_{i-1, j} .
$$

Since $j-1 \stackrel{4}{\equiv} 0$, we obtain

$$
(L \cdot U)_{i-1, j-1}=l_{i-1,1}-5 \sum_{k=1}^{\frac{j-1}{4}} l_{i-1,4 k}-\sum_{k=1}^{\frac{j-5}{4}} l_{i-1,4 k+1}-\sum_{k=0}^{\frac{j-5}{4}} l_{i-1,4 k+2}+3 \sum_{k=0}^{\frac{j-5}{4}} l_{i-1,4 k+3} .
$$


Similarly, since $j \stackrel{4}{\equiv} 1$ it follows that

$(L \cdot U)_{i-1, j}=l_{i-1,1}+\sum_{k=1}^{\frac{j-1}{4}} l_{i-1,4 k}-5 \sum_{k=1}^{\frac{j-1}{4}} l_{i-1,4 k+1}-\sum_{k=0}^{\frac{j-5}{4}} l_{i-1,4 k+2}-\sum_{k=0}^{\frac{j-5}{4}} l_{i-1,4 k+3}$.

Therefore, we have

$$
(L \cdot U)_{i-1, j-1}-(L \cdot U)_{i-1, j}=-6 \sum_{k=1}^{\frac{j-4}{4}} l_{i-1,4 k}+4 \sum_{k=1}^{\frac{j-5}{4}} l_{i-1,4 k+1}+4 \sum_{k=0}^{\frac{j-5}{4}} l_{i-1,4 k+3}-5 l_{i-1, j} .
$$

Furthermore, since $j \stackrel{4}{\equiv} 1$ we obtain

$$
(L \cdot U)_{i, j}=l_{i, 1}+\sum_{k=1}^{\frac{j-1}{4}} l_{i, 4 k}-5 \sum_{k=1}^{\frac{j-1}{4}} l_{i, 4 k+1}-\sum_{k=0}^{\frac{j-5}{4}} l_{i, 4 k+2}+\sum_{k=0}^{\frac{j-5}{4}} l_{i, 4 k+3} .
$$

Now we apply (11), to get

$$
\begin{aligned}
(L \cdot U)_{i, j}=1 & +\sum_{k=1}^{\frac{j-1}{4}}\left(l_{i-1,4 k-1}-l_{i-1,4 k}\right)-5 \sum_{k=1}^{\frac{j-4}{4}}\left(l_{i-1,4 k}-l_{i-1,4 k+1}\right) \\
& -\sum_{k=0}^{\frac{j-5}{4}}\left(l_{i-1,4 k+1}+l_{i-1,4 k+2}\right)+\sum_{k=0}^{\frac{j-5}{4}}\left(l_{i-1,4 k+2}+l_{i-1,4 k+3}\right) .
\end{aligned}
$$

After some simplifications this leads to

$$
(L \cdot U)_{i, j}=-6 \sum_{k=1}^{\frac{j-4}{4}} l_{i-1,4 k}+4 \sum_{k=1}^{\frac{j-5}{4}} l_{i-1,4 k+1}+4 \sum_{k=0}^{\frac{j-5}{4}} l_{i-1,4 k+3}-5 l_{i-1, j} .
$$

Through comparing (19) and (20), we can get (18).

Case $3 . j \stackrel{4}{\equiv} 2$. In this case we claim that

$$
(L \cdot U)_{i, j}=(L \cdot U)_{i-1, j-1}+(L \cdot U)_{i-1, j} .
$$

Here since $j-1 \stackrel{4}{\equiv} 1$, by (12) we obtain

$$
(L \cdot U)_{i-1, j-1}=1+\sum_{k=1}^{\frac{j-2}{4}} l_{i-1,4 k}-5 \sum_{k=1}^{\frac{j-2}{4}} l_{i-1,4 k+1}-\sum_{k=0}^{\frac{j-6}{4}} l_{i-1,4 k+2}+\sum_{k=0}^{\frac{j-6}{4}} l_{i-1,4 k+3},
$$

and since $j \stackrel{4}{\equiv} 2$ it follows that

$$
(L \cdot U)_{i-1, j}=1+\sum_{k=1}^{\frac{j-2}{4}} l_{i-1,4 k}+3 \sum_{k=1}^{\frac{j-2}{4}} l_{i-1,4 k+1}+\sum_{k=0}^{\frac{j-2}{4}} l_{i-1,4 k+2}-\sum_{k=0}^{\frac{j-6}{4}} l_{i-1,4 k+3} .
$$


Therefore we have

(22) $(L \cdot U)_{i-1, j-1}+(L \cdot U)_{i-1, j}=2+2 \sum_{k=1}^{\frac{j-2}{4}} l_{i-1,4 k}-2 \sum_{k=1}^{\frac{j-2}{4}} l_{i-1,4 k+1}+l_{i-1, j}$.

On the other hand, since $j \stackrel{4}{\equiv} 2$ we deduce that

$$
(L \cdot U)_{i, j}=1+\sum_{k=1}^{\frac{j-2}{4}} l_{i, 4 k}+3 \sum_{k=1}^{\frac{j-2}{4}} l_{i, 4 k+1}+\sum_{k=0}^{\frac{j-2}{4}} l_{i, 4 k+2}-\sum_{k=0}^{\frac{j-6}{4}} l_{i, 4 k+3},
$$

and by (11) we conclude that

$$
\begin{aligned}
(L \cdot U)_{i, j}=1 & +\sum_{k=1}^{\frac{j-2}{4}}\left(l_{i-1,4 k-1}-l_{i-1,4 k}\right)+3 \sum_{k=1}^{\frac{j-2}{4}}\left(l_{i-1,4 k}-l_{i-1,4 k+1}\right) \\
& +\sum_{k=0}^{\frac{j-2}{4}}\left(l_{i-1,4 k+1}+l_{i-1,4 k+2}\right)-\sum_{k=0}^{\frac{j-6}{4}}\left(l_{i-1,4 k+2}+l_{i-1,4 k+3}\right) .
\end{aligned}
$$

Now, an easy calculation shows that

$$
(L \cdot U)_{i, j}=2+2 \sum_{k=1}^{\frac{j-2}{4}} l_{i-1,4 k}-2 \sum_{k=1}^{\frac{j-2}{4}} l_{i-1,4 k+1}+l_{i-1, j} .
$$

By comparing (22) and (23), we may obtain (21).

Case 4 . $j \stackrel{4}{\equiv} 3$. In this case, we claim that

$$
(L \cdot U)_{i, j}=(L \cdot U)_{i-1, j-1}+(L \cdot U)_{i-1, j} .
$$

Since $j-1 \stackrel{4}{\equiv} 2$, we obtain

$$
(L \cdot U)_{i-1, j-1}=1+\sum_{k=1}^{\frac{j-3}{4}} l_{i-1,4 k}+3 \sum_{k=1}^{\frac{j-3}{4}} l_{i-1,4 k+1}+\sum_{k=0}^{\frac{j-3}{4}} l_{i-1,4 k+2}-\sum_{k=0}^{\frac{j-7}{4}} l_{i-1,4 k+3} .
$$

Similarly, since $j \stackrel{4}{\equiv} 3$ it follows that

$$
(L \cdot U)_{i-1, j}=1-\sum_{k=1}^{\frac{j-3}{4}} l_{i-1,4 k}-\sum_{k=1}^{\frac{j-3}{4}} l_{i-1,4 k+1}-\sum_{k=0}^{\frac{j-3}{4}} l_{i-1,4 k+2}+\sum_{k=0}^{\frac{j-3}{4}} l_{i-1,4 k+3} .
$$

Therefore, we have

(25) $(L \cdot U)_{i-1, j-1}+(L \cdot U)_{i-1, j}=2+2 \sum_{k=1}^{\frac{j-3}{4}} l_{i-1,4 k+1}+2 \sum_{k=0}^{\frac{j-3}{4}} l_{i-1,4 k+2}+l_{i-1, j}$. 
On the other hand, since $j \stackrel{4}{\equiv} 2$ we obtain

$$
(L \cdot U)_{i, j}=1-\sum_{k=1}^{\frac{j-3}{4}} l_{i, 4 k}-\sum_{k=1}^{\frac{j-3}{4}} l_{i, 4 k+1}+\sum_{k=0}^{\frac{j-3}{4}} l_{i, 4 k+2}-\sum_{k=0}^{\frac{j-3}{4}} l_{i, 4 k+3} .
$$

Again by (11) we conclude that

$$
\begin{aligned}
(L \cdot U)_{i, j}= & 1-\sum_{k=1}^{\frac{j-3}{4}}\left(l_{i-1,4 k-1}-l_{i-1,4 k}\right)-\sum_{k=1}^{\frac{j-3}{4}}\left(l_{i-1,4 k}-l_{i-1,4 k+1}\right) \\
& +\sum_{k=0}^{\frac{j-3}{4}}\left(l_{i-1,4 k+1}+l_{i-1,4 k+2}\right)+\sum_{k=0}^{\frac{j-3}{4}}\left(l_{i-1,4 k+2}+l_{i-1,4 k+3}\right),
\end{aligned}
$$

and we easily deduce that

$$
(L \cdot U)_{i, j}=2+2 \sum_{k=1}^{\frac{j-3}{4}} l_{i-1,4 k+1}+2 \sum_{k=0}^{\frac{j-3}{4}} l_{i-1,4 k+2}+l_{i-1, j} .
$$

By comparing (25) and (26), we can get (24).

Therefore, from (13), (14), (15), (18), (21) and (24) we conclude the theorem.

In the end, we consider the sequence $\left(\operatorname{det} A_{\alpha, \alpha}^{(3, \pm)}(n)\right)$ for $n \in \mathbb{N}$.

Theorem 3. The sequence $\left(\operatorname{det} A_{\alpha, \alpha}^{(3, \pm)}(n)\right)$ of determinants associated to the sequence $\alpha=(1,1,1, \ldots)$ satisfies the following

$$
\operatorname{det} A_{\alpha, \alpha}^{(3, \pm)}(n)= \begin{cases}11^{3 l-1} & \text { if } n=6 l, \quad(l=1,2, \ldots) \\ 11^{3 l} & \text { if } n=6 l+r,(r=1,2,3,4, l=0,1,2, \ldots) \\ 11^{3 l+1} & \text { if } n=6 l+5 .(l=0,1,2, \ldots)\end{cases}
$$

Proof. The proof is similar to the proof of Theorem 1.1 and Theorem 1.2, and we avoid presenting some of the details. Again, we apply LU-factorization. Here, we claim that

$$
A_{\alpha, \alpha}^{(3, \pm)}(n)=L \cdot U
$$

where $L=A_{\beta, \alpha}^{(3, \pm)}(n)$ with $\beta=(1,0,0, \ldots)$ is a lower triangular matrix with 1 's on the diagonal, and where

$$
U=\left[\begin{array}{c}
U_{1} \\
U_{2} \\
\vdots \\
U_{n}
\end{array}\right]
$$


with

$$
U_{i}= \begin{cases}\underbrace{(1,1,1, \ldots, 1}_{n \text { times }}) & i=1, \\ \underbrace{0,0, \ldots, 0}_{i-1 \text { times }}, \underbrace{11,-7,1,1,-1,-1, \ldots, u_{i, n-1}, u_{i, n}}_{n-i+1 \text { times }(6-\text { periodic })}) & i \stackrel{6}{\equiv} 0, \\ (\underbrace{0,0, \ldots, 0}_{i-1 \text { times }}, \underbrace{11,-5,3,-1,-1,-1, \ldots, u_{i, n-1}, u_{i, n}}_{n-i+1 \text { times }(6-\text { periodic })}) & i \stackrel{6}{\equiv} 1, \\ (\underbrace{0,0, \ldots, 0}_{i-1 \text { times }}, \underbrace{1,1,1,-1,-1,-1, \ldots, u_{i, n-1}, u_{i, n}}_{n-i+1 \text { times }(6-\text { periodic })}) & i \stackrel{6}{=} 2, \underbrace{1,1,3,1,1,-1, \ldots, u_{i, n-1}, u_{i, n}}_{n-i+1 \text { times }(6-\text { periodic })}) \\ (\underbrace{0,0, \ldots, 0}_{i-1 \text { times }}, \underbrace{1,-5,1,-1,1,-1, \ldots, u_{i, n-1}, u_{i, n}}_{n-i+1 \text { times }(6-\text { periodic })}) & i \stackrel{6}{\equiv} 4, \\ (\underbrace{0,0, \ldots, 0}_{i-1 \text { times }}, \underbrace{11,-7,3,-1,1,-1, \ldots, u_{i, n-1}, u_{i, n}}_{n-i+1 \text { times }(6-\text { periodic })}) & i \stackrel{6}{\equiv} 5,\end{cases}
$$

and $\left(u_{i, n-1}, u_{i, n}\right)$ is satisfied in Table 3 .

Table 3.

\begin{tabular}{|c|c|c|c|c|c|c|}
\hline$i \backslash n$ & $n \stackrel{6}{\equiv} 0$ & $n \stackrel{6}{\equiv} 1$ & $n \stackrel{6}{\equiv} 2$ & $n \stackrel{6}{\equiv} 3$ & $n \stackrel{6}{\equiv} 4$ & $n \stackrel{6}{\equiv} 5$ \\
\hline$i \stackrel{6}{\equiv} 0$ & $(-1,11)$ & $(11,-7)$ & $(-7,1)$ & $(1,1)$ & $(1,-1)$ & $(-1,-1)$ \\
\hline$i \stackrel{6}{\equiv} 1$ & $(-1,-1)$ & $(-1,11)$ & $(11,-5)$ & $(-5,3)$ & $(3,-1)$ & $(-1,-1)$ \\
\hline$i \stackrel{6}{\equiv} 2$ & $(-1,-1)$ & $(-1,-1)$ & $(-1,1)$ & $(1,1)$ & $(1,1)$ & $(1,-1)$ \\
\hline$i \stackrel{6}{\equiv} 3$ & $(3,1)$ & $(1,1)$ & $(1,-1)$ & $(-1,1)$ & $(1,1)$ & $(1,3)$ \\
\hline$i \stackrel{6}{\equiv} 4$ & $(-5,1)$ & $(1,-1)$ & $(-1,1)$ & $(1,-1)$ & $(-1,1)$ & $(1,5)$ \\
\hline$i \stackrel{6}{\equiv} 5$ & $(11,-7)$ & $(-7,3)$ & $(3,-1)$ & $(-1,1)$ & $(1,-1)$ & $(-1,11)$ \\
\hline
\end{tabular}

The matrix $U$ is an upper triangular one with diagonal entries

$$
1, \underbrace{1,1,1,11,11,11, \ldots, u_{n-1, n-1}, u_{n, n}}_{6-\text { periodic }},
$$

where

$$
\left(u_{n-1, n-1}, u_{n, n}\right)= \begin{cases}(11,11) & \text { if } n \stackrel{6}{\equiv} 0 \text { or } 1 \\ (11,1) & \text { if } n \stackrel{6}{\equiv} 2 \\ (1,1) & \text { if } n \stackrel{6}{\equiv} 3 \text { or } 4 \\ (1,11) & \text { if } n \stackrel{6}{\equiv} 5\end{cases}
$$


Since $\operatorname{det} L=1$ and

$$
\operatorname{det} U= \begin{cases}11^{3 l-1} & \text { if } \quad n=6 l, \quad(l=1,2, \ldots) \\ 11^{3 l} & \text { if } \quad n=6 l+r,(r=1,2,3,4, l=0,1,2, \ldots) \\ 11^{3 l+1} & \text { if } \quad n=6 l+5,(l=0,1,2, \ldots)\end{cases}
$$

it is obvious that the claimed factorization of $A_{\alpha, \alpha}^{(3, \pm)}(n)$ implies the validity of the theorem.

Let us do the required calculation. Again, we assume that

$$
L=\left(l_{i, j}\right)_{1 \leq i, j \leq n} \quad \text { and } \quad U=\left(u_{i, j}\right)_{1 \leq i, j \leq n} .
$$

Then by definition, we have $l_{1,1}=1, l_{1, j}=0, l_{i, 1}=1$ for $2 \leq i, j \leq n$ and the entries $l_{i, j}$ for $2 \leq i, j \leq n$ satisfy

$$
l_{i, j}= \begin{cases}l_{i-1, j-1}+l_{i-1, j} & \text { if } j \stackrel{4}{=} 2,3,4 \\ l_{i-1, j-1}-l_{i-1, j} & \text { if } j \stackrel{4}{=} 5,0,1 .\end{cases}
$$

Moreover, the $j$ th column of $U$ can be considered as follows.

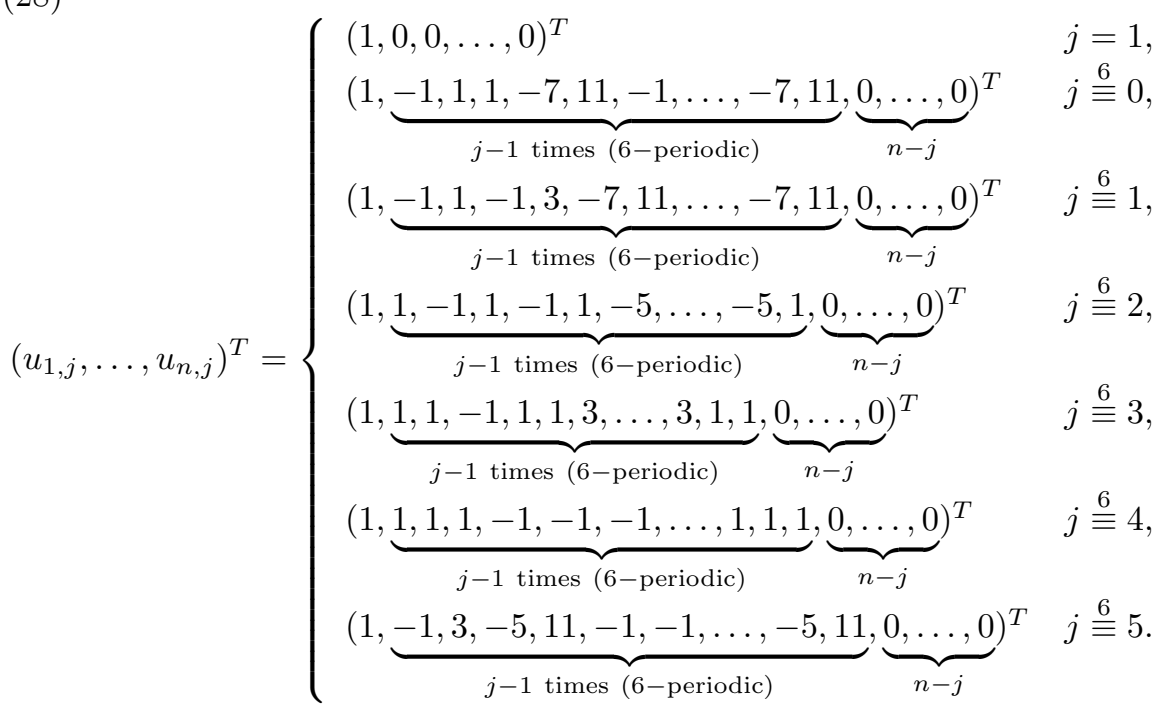

In order to prove the claim we show that the $(i, j)$-entry of $L \cdot U$, that is

$$
(L \cdot U)_{i, j}=\sum_{k=1}^{n} l_{i, k} u_{k, j}
$$

satisfy $(L \cdot U)_{1, j}=1,(L \cdot U)_{i, 1}=1$ for $1 \leq i, j \leq n$ and

$$
(L \cdot U)_{i, j}= \begin{cases}(L \cdot U)_{i-1, j-1}+(L \cdot U)_{i-1, j} & j \stackrel{6}{\equiv} 2,3,4 \\ (L \cdot U)_{i-1, j-1}-(L \cdot U)_{i-1, j} & j \stackrel{6}{\equiv} 5,0,1\end{cases}
$$

for $2 \leq i, j \leq n$. 
First assume that $i=1$. Then, in accordance with the definition of $l_{1, j}$, we obtain

$$
(L \cdot U)_{1, j}=\sum_{k=1}^{n} l_{1, k} u_{k, j}=l_{1,1} u_{1, j}=1 .
$$

Next, suppose that $j=1$. In this case by (28) we obtain

$$
(L \cdot U)_{i, 1}=\sum_{k=1}^{n} l_{i, k} u_{k, 1}=l_{i, 1} u_{1,1}=1
$$

Finally, we assume that $2 \leq i, j \leq n$. In this case we must show that the entries $(L \cdot U)_{i, j}$ satisfy (29). Here, there are six cases to distinguish, according to $j \stackrel{6}{\equiv} 0,1,2,3,4$ or 5 . Using similar arguments to those in the proof of Theorem 1.2, we see that the result is true in any cases. For instance, we assume that $j \stackrel{6}{\equiv} 4$. In this case, we must establish that

$$
(L \cdot U)_{i, j}=(L \cdot U)_{i-1, j-1}+(L \cdot U)_{i-1, j}
$$

Since $j \stackrel{6}{\equiv} 4$, in according with $(28)$, the right hand of (30) is equal to

$$
2+2 \sum_{k=1}^{\frac{j-4}{6}} l_{i-1,6 k+1}+2 \sum_{k=0}^{\frac{j-4}{6}} l_{i-1,6 k+2}+2 \sum_{k=0}^{\frac{j-4}{6}} l_{i-1,6 k+3}+l_{i-1, j}
$$

Again, since $j \stackrel{4}{\equiv} 2$ by (24), we see that the left-hand of (30) is equal to

(32) $1-\sum_{k=1}^{\frac{j-4}{6}} l_{i, 6 k}-\sum_{k=1}^{\frac{j-4}{6}} l_{i, 6 k+1}+\sum_{k=0}^{\frac{j-4}{6}} l_{i, 6 k+2}+\sum_{k=0}^{\frac{j-4}{6}} l_{i, 6 k+3}+\sum_{k=0}^{\frac{j-4}{6}} l_{i, 6 k+4}-\sum_{k=0}^{\frac{j-10}{6}} l_{i, 6 k+5}$.

Now, if we substitute the corresponding value for $l_{i, 6 k+r}(0 \leq r \leq 5)$ from (29), we can conclude

$$
(L \cdot U)_{i, j}=2+2 \sum_{k=1}^{\frac{j-4}{6}} l_{i-1,6 k+1}+2 \sum_{k=0}^{\frac{j-4}{6}} l_{i-1,6 k+2}+2 \sum_{k=0}^{\frac{j-4}{6}} l_{i-1,6 k+3}+l_{i-1, j}
$$

which results in (30). In this way the proof is completed.

\section{References}

[1] R. Bacher, Determinants of matrices related to the Pascal triangle, J. Theor. Nombres Bordeaux 14 (2002), no. 1, 19-41.

[2] C. Krattenthaler, Advanced determinant calculus, Sem. Lothar. Combin. 42 (1999), Art. B42q, 67 pp.

[3] _ Evaluations of some determinants of matrices related to the Pascal triangle, Sem. Lothar. Combin. 47 (2001/02), Art. B47g, 19 pp. 
Ali Reza Moghaddamfar

Department of Mathematics

FACUlty OF SCIENCE

K. N. Toosi University of Technology

P. O. Box 16315-1618, TEHran, IraN

AND

SChOOL OF MATHEMATiCS

Institute for Studies in Theoretical Physics and Mathematics (IPM)

P. O. Box 19395-5746, Theran, IrAN

E-mail address: moghadam@kntu.ac.ir and moghadam@mail.ipm.ir

SEYYed NAVID SALEHY

Department of Mathematics

FACUlty OF SCIENCE

K. N. Toosi University of Technology

P. O. Box 16315-1618, TEHRAN, IrAN

Seyyed Nima Salehy

Department of Mathematics

FACUlty OF SCIENCE

K. N. Toosi University of Technology

P. O. Box 16315-1618, Tehran, IraN 